\title{
The application status and development trend of sports statistical methods
}

Long Lin

Guangzhou Sport University,Guangzhou,Guangdong,510500,Guangzhou,China.

Abstract: The continuous and in-depth development of the reform of the education system and the changes in science and technology have gradually made the methods of sports research by the majority of educators become more diverse. Therefore, while achieving high achievements in sports statistics research, physical education teachers in colleges and universities need to be able to use them efficiently in order to achieve the purpose of establishing high-quality sports classrooms. This article starts with the actual situation of the application of sports statistics and discusses its main development trends, hoping to provide some reference for other educators.

Keywords: Physical Education Class; Development Trend; Higher Education; Sports Statistics Method

As an important cradle for cultivating skilled and high-quality talents, higher education often requires educators to find ways to effectively establish high-efficiency classroom teaching through continuous innovation and research. Therefore, many sports educators continue to explore in the scientific field and introduce sports statistics methods into actual scientific research, in order to achieve the goal of completing high-efficiency sports statistics. This requires that physical education teachers in colleges and universities should strengthen the application of sports statistical methods, and be able to combine statistical methods and models with high quality based on the current actual situation, so that sports statistical methods can be used effectively while ensuring overall education High-quality work carried out.

\section{The Reality of Sports Statistics Application}

\subsection{High acceptance}

In the current era of rapid changes in science and technology, sports statistical methods have gradually received more and more attention, and sports statistical methods will be seen in almost all data analysis fields. Therefore, whether it is the existing scientific research results of colleges and universities or the fast-developing sports competitions, the high-quality use of sports statistical methods will help people use sample content analysis to conduct in-depth research on total parameters more accurately. Therefore, under the current situation where the acceptance of sports statistical methods is very high, it can not only help scientific research results become more accurate, but also can effectively improve the quality of statistics while greatly reducing economic capital investment. In addition, the scope of application of sports statistics is very wide, and it is widely involved in current sports medicine, sports biology and other disciplines, so it is worthy of extensive attention and application by educators.

\subsection{Solid academic status}

After more than 40 years of long research, the current sports statistics method has gradually become a more complete subject knowledge system with the continuous efforts of all sports educators. Therefore, in order to enable sports statistics methods to contribute more value to the field of sports education, many colleges and universities will incorporate sports statistics into the construction of key curriculum systems when designing sports majors. Therefore, Copyright (C) 2020 Long Lin

doi: 10.18282/le.v9i5.1214

This is an open-access article distributed under the terms of the Creative Commons Attribution Non-Commercial License

(http://creativecommons.org/licenses/by-nc/4.0/), which permits unrestricted non-commercial use, distribution, and reproduction in any medium, provided the original work is properly cited. 
with the gradual consolidation of the status of sports statistics itself, it is more necessary to ensure the smooth progress of its teaching work. The sports statistics subject has a wide range of content, mainly including correlation analysis, variance analysis, hypothesis testing, regression analysis, parameter estimation, correlation analysis, multivariate analysis, etc., which can enable students to learn knowledge systematically and through the use of sports statistical methods, Good access to data to ensure better development of statistical activities.

\section{The future development direction of sports statistics in colleges and universities}

\subsection{Pay attention to statistical foundation}

Since sports statistics have formed a relatively complete system, when using sports statistics methods in colleges and universities, educators should not simply use them as calculation tools, but should objectively analyze the data to improve the statistical results in a targeted manner. Sports statistical methods take classic statistics as an important basis for their own development, so they can effectively process independent data with high quality. With the development of the times and the continuous advancement of technology, sports data presents more diverse forms, and incomplete threedimensional data and nonlinear data are derived. Therefore, the future development direction of sports statistics should be based on the basis of statistics, combined with related practical activities, and cross-integrated with other disciplines, in order to ensure the rapid improvement of the efficiency of statistical analysis. As the current statistical methods are more and more closely related to Internet technology, the support of statistical methods is required for some data feature measurement and detection processes and mathematical model establishment. Therefore, educators in higher education institutions need to pay more attention to theoretical foundations in order to find more efficient statistical methods based on actual conditions, and to ensure the smooth completion of statistical work.

\subsection{Improve the sports statistical method system}

In order to ensure that sports statistical methods can achieve more long-term development in the context of constant changes. Therefore, educators need to conduct an in-depth analysis of the basic framework of the current sports statistical methods, and be able to continuously innovate the attributes and disciplinary characteristics of sports itself, and through the breakthrough of the old statistical system, find better innovations under the blessing of emerging technologies. way. Therefore, sports statisticians need to take the improvement of the sports statistics method system as the important content of the future development direction, and the smooth development of practical activities as the sound basis, analyze the actual data cases of the sports profession, and construct a more comprehensive Method system. In addition, with the rapid development of statistics and sports, it is necessary for educators to conduct in-depth analysis of the problems in current sports statistics methods in a timely manner, and actively integrate the development of the times into AHP, analysis, data mining, and fuzzy mathematics. And other emerging methods, to ensure that the sports statistical method system is constantly improving, while achieving better development.

\section{Concluding remarks}

In order to promote the long-term development of the physical education industry, after many years, with the unremitting efforts of statisticians, more and more educators pay attention to the method of sports statistics. With the deepening of the reform of the education system, more and more physical education teachers pay attention to the use of sports statistics methods. Therefore, it is necessary for educators to analyze the current use of sports statistical methods and future development trends in the context of continuous discovery of the times, so as to make better use of statistical methods and ensure the better development of physical education.

\section{References}

1. Wang Chao, Guo Jing. The teaching mode of "Sports Statistics" based on improving students' practical ability and the 
reform of evaluation method under the framework of VBA+Excel [C]. Chinese Sports Science Association. The 11th National Sports Science Abstract compilation of conference papers. Chinese Society of Sports Science, 2019: 58035804.

2. Zheng Kai. Research on the application status and development trend of sports statistical methods [C]. China Sport Science Society. Collection of abstracts of the 2015 Tenth National Sports Science Conference (2). China Sport Science Society (China Sport Science Society): China Sport Science Society, 2015: 955-956.

3. Liu Wei, Zheng Zhiqiang. Sports scientific research as an art and statistical methods as a tool— Some views on statistical methods in sports scientific research [J]. Journal of Tianjin Institute of Physical Education, 2001(04): 3741. 